changes are unquestionably due to increased amount of oxygen inspired. Anemic conditions rapidly disappear in the sunlight of Mexico's high altitudes; rapid and remarkable improvement in the local and general conditions are coincident with the blood changes in the consumptive who is transported from the sea level to altitudes of three or more thousand feet, as has been demonstrated by numerous careful observations in Europe, North and South America.

So far as we know the only successful means of combating tuberculosis is by improving the resisting powers of the system. Out-door life in a mild, dry climate where the general health can be kept at the highest possible standard offers certainly the best possible advantages and the longest immunity. The high lands of Mexico certainly offer all that can be desired in point of climate, being neither hot nor cold, and is as near as possible suited to each and all cases. Whatever rainfall takes place in Mexico is between the months of April and November, just when needed to cool the otherwise long and occasionally dry season, most of the showers occurring during the night hours consequently seldom interferes with out-door exercise, sports or employment. The opportunity for profitable employment is a strong argument in favor of Mexico and New Mexico; lands are cheap in either country and profits large. The accommodations for invalids are meager. The hotels are not, as a rule, what they ought to be and are not generally favorably located for the invalid class, nor are they built with any thought of providing open air exercise or sun baths. The hotels are in the midst of the noise and din of business. That no provision has been made for the invalid class is due to lack of demand; the people of the North that need just such a climate have never had their attention called to Mexico. The day is near at hand when the sanitariums for the people of the more northern sections will be in the Mexicos. The land of the Montezumas needs only to be seen to be appreciated, the enchantment grows with your stay in that balmy land. While there are English-speaking people to be found almost everywhere in the republic and especially in the City of Mexico, your comfort will be largely increased if you have sufficient knowledge of the Spanish language to understand and be understood.

\section{CLASSIFICATION OF THE LESIONS CONSTI- TUTING THE SO-CALLED CHRONIC NASAL CATARRH.}

Read before the Chicago Medical society, Jan, 5, 1898. BY H. GRADLE, M.D.

CHICAGO, ILL.

The term chronic catarrh was formerly used for all chronic affections of the nose manifested by more or less nasal obstruction and secretion. It is still so employed by the public and to some extent by physicians, yet the studies of the last twenty years have resolved this comprehensive name into a number of separate affections. The description of these various types of disease is, however, neither uniform in different text-books on the nose, nor do the types stated by authors conform always with actual observation. Perhaps the main reason for these discrepancies is the frequent multiplicity of lesions, so that quite commonly the symptoms of catarrh depend on several different morbid conditions co-existing in the nose.

My object is to classify the different lesions which make up this composite picture of catarrh. On the basis of many years' personal observation I wish to describe those types of morbid changes which constitute each a separate disease entity. Although these are often found in combination I shall base my description on cases which present the least number of lesions and hence the purest type of each disease.

The different lesions which simply or in combination make up the clinical picture of chronic catarrh may be grouped as follows: 1, enlargement of cavernous tissue; 2, simple nasal catarrh ; 3 , nasal suppuration, localized in nasal passages, accessory cavities, or the pharynx; $a$, rhinitis sicca anterior (perforating ulcer); b, ozena or atrophic rhinitis; 4, hypertrophic inflammation of mucous membrane and adenoid tissue; 5 , septum deformities in the form of deflection, articular expansion or callus formation.

1. Enlargement of the renous plexus in the mucous membrane occurs in connection with all (except the atrophic) forms of nasal disease, but may exist in a nose not otherwise diseased at the time. It hence deserves to be described as a separate lesion. Its significance depends less on the permanent increase of vascular tissue than on its periodic dilatation under the infiuence of various stimuli. These periodic changes of turgescence cause the variable obstruction or even occlusion in nasal diseases. Except in acute inflammations the vascular distension is only onesided at a time; but alternating. The area where the veins are massed in the form of distinct cavernous tissue is the front region of the inferior turbinal and the posterior ends of all the turbinals. Enlargement of the cavernous tissue is not identical with hypertro. phy of the mucous membrane and can be distin. guished from it by the compressibility on pressure with the probe and by the contraction under the influ. ence of cocain. It may, however, co-exist with moderate tissue hypertrophy.

The influence of the vaso-constrictor nerves upon the cavernous tissue is sometimes shown by its con. traction under the effect of anxiety during an examination. Whether there are also vaso-dilator nerves is not known. Although enlargement of the cavernous bodies is mostly but a concomitant condition in other nasal diseases, yet it is sometimes found where no other lesion can be detected, especially in hay fever. As far as can be learned clinically, it is the result of often repeated attacks of acute inflammation in sub. jects with unstable nervous systems. Indeed vascular dilatability is the direct expression and, to some extent, a proportionate index of a neuro-pathic condi. tion. In extreme instances sudden engorgement with sneezing and profuse watery flow simulates attacks of true coryza, but its non-inflammatory nature is shown by the short duration of the spell, as well as by the absence of corpuscles in the clear discharge.

2. True nasal catar $\cdot h$ is characterized by catarrhal secretion, a thick, viscid, turbid fluid, containing enough cells to make it appear whitish or gray, but not yellow or green and not at all purulent. In cities the discharge is commonly stained by inhaled soot and dust. This discharge flows over the soft palate and drops into the throat or is dislodged by forcible expirations which vibrate the palate. It is not blown out of the nose. Nasal discharge blown in to the handkerchief is either purulent or a sudden flow of clear transparent mucus in consequence of acute irritation. Although nasal catarrh, described by the patient as a "dropping in the throat," is the most common form of 
nasal disease in this climate, I do not know of any accurate description of it in literature. It is usually classified as chronic hypertrophic catarrh, but the description does not conform to the reality. Various forms of hypertrophy frequently occur with true catarrh, but they are not a constant and hence not an integral factor. In uncomplicated cases of true catarrh there are no gross lesions visible to the eye. Yet I do not know of any author who has defined chronic nasal catarrh in this manner.

What, then, is the pathology of simple chronic catarrh? In those few instances in which it can be traced from its beginning, it is found to be the sequel of an acute coryza. Moreover, it is always intensified when the patient passes through a fresh acute nasal inflammation. This history suggests that it is due to a persistence of chronic inflammation. As far as can be learned clinically, the process is localized in the posterior and lower part of the nose. But in the pure type of the clisease the inflammation does not reveal itself by either redness or swelling of the mucous membrane, at least as seen during life by anterior or posterior rhinoscopy. In view of the fact that the catarrhal secretion persists in some patients for many years without being followed by any visible tissue alterations, the term hypertrophic catarrh does not seem appropriate. Moreover, the hypertrophic areas which are often found associated with simple catarrh are not necessarily the source of any secretion.

Bosworth has emphasized that an important factor in nasal catarrh is stenosis of the nasal passage, especially when due to irregularity of the septum. The amount of catarrhal secretion and the annoyance to which it gives rise are more or less proportionate to the narrowness of one or both sides of the nose. But I can not accept Bosworth's view, that the secretion is the direct consequence of the stenosis. Stenosis of variable degree may exist by itself without nasal catarrh or secretion, especially in young persons under about 16 years of age, in whom nasal catarrh is quite rare. Adults, however, who have nasal stenosis, quite commonly acquire catarrh in addition, which proves persistent. On the other hand, I have observed chronic catarrh in noses which were not at all stenotic. The only method of estimating nasal stenosis is to listen to the sound produced by breathing through one side at a time. A normal passage allows the air to pass in and out without any sound whatever, while interference with respiration gives rise to a noise which varies from a gentle blowing to a sharp hiss. I regard stenosis as an important determining condition, which favors the persistence of chronic inflammation in the posterior parts of the nose, but not as an indispensable factor in catarrh. All operative procedures against stenosis relieve the catarrh in proportion to their mechanical influence upon the caliber of the nasal passage. Of similar importance are the transient occlusions due to vascular engorgement; hence removal of excessive cavernous tissue by snare or cautery is a valuable remedial measure in suitable cases.

The surgical cure of stenosis does not cure the catarrh. The secretion, though much diminished, still persists. As the patient feels very little annoyance from this when the nose is freely patent, he is generally satisfied, but the catarrhal secretion is there. 1 know of no method by which we can absolutely cure chronic nasal catarrh; it may however disappear in the course of time or under favorable climatic influ- ences in noses without stenosis, or after successful operation for stenosis.

3. Nusal :upunution is separate and distinct from nasal catarrh, Its secretion is pus diluted with more or less mucus, and chronic pyogenic infection always involves a possibility of mischief by extension. The source of the pus may be in the nasal passages, in the accessory sinuses or in the pharynx. Gruenwald has shown chinically that clironic suppurative inflammation in the nose is usually not a diffuse process, but localized in some small area. In many of his cases he claims to have found caries or superficial ulceration of the bone as the lesion which explained the persistent suppuration. I have seen very little confirmation of this part of Gruenwald's view and I do not think that the information which he gets from the use of the probe is as trustworthy, regarding bone disease in hidden parts of the nose, as he claims. I also do not admit the existence of Woakes' necrosing ethmoiditis as a common lesion in nasal suppuration. My doubts are fully confirmed by the recent pathologic studies of J. N. Mackenzie and of Hajek. In seventeen amputated fragments of the middle turbinal, in the class of case attributed by Woakes to necrosis, Hajek did not find bone destruction, anatomically, in a single instance. Destruction of bone does, however, occur in some of the more severe forms of suppuration of the accessory cavities, but such cases are relatively rare. The more common cause of osseous necrosis is syphilis in its late manifestations. A purulent dis. charge may also be due to foreign bodies and concretions in the nasal passages. Another cause for the persistence of purulent inflammation is the lodging of pus in various recess-shaped areas and in such regions where accidental configuration favors a damming up of pus; by reason of such imperfect drainage, acute attacks of coryza lead to the persistence of localized suppurative inflammation.

While disease of the accessory sinuses was considered scarcely more than a surgical curiosity until some ten years ago, anatomic work by Harke, E. Fraenkel and others has shown the frequency of acute affections at autopsies. Probably most of the milder acute cases escape clinical detection and heal spontaneously, but the more our methods of diagnosis become perfected, the larger do we find the proportion of diseased nasal accessory cavities as the source of nasal suppuration. It is beyond the scope of this paper to discuss the methods of diagnosis and treatment. While the more severe "text-book" cases of sinus suppuration can now be readily recognized, it often taxes the patience and ingenuity to determine the source of pus in the less pronounced forms. In the milder forms of even chronic suppuration from the frontal, ethmoid or sphenoid sinus, a cure can sometimes be obtained without surgical exposure of these cavities, if by minor surgical intranasal meas. ures we can improve the drainage.

Pus secreted in the nose, the ethmoid cells or the sphenoid sinus may flow into the pharynx, as well as out of the nose anteriorly, where the configuration of the passages favors the flow toward the rear, but pus in the pharynx may also be formed in this space itself. Its source, in such cases, is the inflamed adenoid tissue at the roof, the pharyngeal tonsil. Mere enlargement of the adenoid tissue does not necessarily cause suppuration; the tissue must be besides in a state of intlammation. An enlarged pharyngeal tonsil does not suppurate continuously, although it does 
so very often. On the other hand, there may be purulent inflammation at the pharyngeal vault without enough hypertrophy to cause the symptoms of obstruction.

In certain cases the morbid discharge formed at the roof of the pharynx dries in the form of adherent crusts. As a rule, we find in such instances only a slightly thickened layer of adenoid tissue. Sometimes the secretion issues from clefts in the adenoid tissue, the surface of which appears healthy. Whether some of these instances are not cases of ozena localized in the pharynx, is an open question.

3a. Rhinitis sicca anterior of Sieberman, a special form of nasal suppuration, is a disease limited to the anterior lower part of the surface of the septum. In this affection (usually one sided) the discharge forms a thin adherent scab on the septum, without any odor. Characteristic of the disease is the viscid secretion which, while scant and purulent, dries in so firm a manner that it can not be detached without injury to the epithelium, hence its removal leaves an abraded surface. A further peculiarity is the extensive hyaline degeneration of the involved mucous membrane. (Ribari). This form of disease causes but little subjective annoyance, but it is a frequent source of recurrent nose-bleed. More important, however, is the possibility of ulceration of the septum, for there can be but little doubt that the perforating ulcer of the cartilaginons septum is the occasional consequence of this disease, if not controlled by treatment. This typical form of ulcer has certainly no relation to either tuberculosis or syphilis.

3b. Ozena is another variety of suppurative inflammation of specific character. It is characterized by progressive atrophy of the mucous membrane and turbinate bones, and by a thick purulent secretion drying in crusts, which when allowed to remain give rise to a characteristic fetid odor. The peculiarities of this disease are so pronounced that most observers consider it as a separate affection, but its pathology is not yet clear. The older view, now almost discarded, that it is the terminal stage of hypertrophic inflammation, is unfounded. No one has seen the transformation of a pronounced hypertrophic rhinitis into an atrophic stage. Hypertrophies are common, ozena is relatively infrequent. Moreover, it usually begins in early life. Ozena occurs more commonly in relatively short and wide nasal passages, as Hopman has shown, while hypertrophy is favored by stenosis.

Ozena has been ascribed by Michel, and especially by Gruenwald, to suppuration of accessory cavities, or to circumscribed nasal foci of suppuration. Against this view it can be urged that autopsies have shown that the sinuses are not always involved, but that the greater extent of the mucous membrane is diseased. Undoubtedly sinus suppuration is a frequent and important complication in ozena, but it is only a complication, and one not always present. The microscopic changes in the mucous membrane are, flattening of the epithelial cells and fatty degeneration extending into the mucous glands. The inflammatory nature is shown by round cell infiltration, while fibrillary sclerosis of the mucous membrane leads ultimately to its shrinkage and to atrophy of the bony structures.

The symptoms and course of ozena suggest as its cause some specific microbe. A well characterized bacillus has been described by Loewenberg and studied by Abel, yet in view of the occasional occurrence of this bacillus without ozena, and in the absence of conclusive inoculations, its etiologic role can not be called well established. It is probable that the odor of the discharge is a secondary feature, due to the proneness of this peculiar pus to a certain form of decomposition, for by treatment the odor may be removed even without a complete cure. There is, moreover, a form of atrophic purulent rhinitis identical in course with mild ozena, but without the presence of odor.

4. Hypertrophic inflammation is described separately from simple nasal catarrh, as I do not consider the two affections identical. Many cases of catarrh are not accompanied by any hypertrophy, while hypertrophies are sometimes seen without catarrhal secretion. Moreover, when the two conditions co-exist the areas of hypertrophy are not pecessarily the source of the secretion. We must distinguish between hypertrophies of mucous membrane and of adenoid tissue.

Hypertrophy may involve diffusely the mucous membrane of the inferior and middle turbinal, or may be circumscribed in small areas in the form of tumors. Diffuse hypertrophy can be easily distinguished from mere enlargement of the cavernous tissue by the relative incompressibility and the persistence of the thick cushion after the use of cocain. The hypertrophied membrane is usually pale in color, rarely injected. A peculiar appearance is presented when hypertrophied mucous membrane is edematous; it then appears jelly. like and pellucid. This has been incorrectly termed myxomatous degeneration. The edematous condition, however, is shown by the great shrinkage of excised pieces when placed on blotting paper. Diffuse hypertrophic inflammation gives rise to no symptoms beyond those dependent upon the stenosis which it may produce.

Circumscribed hypertrophies assume various shapes according to their site. On the front end of the inferior and the inferior edge of the middle turbinal they appear as flabby overgrowth. Along the middle of the inferior concha and sometimes on its posterior end the tumors are papillomatous in gross appearance, often resembling raspberries in shape and color. On the posterior ends of inferior and middle turbinals hypertrophies are usually associated with cavernous enlargement and present rounded, grayish, gelatinous looking masses which may seem wrinkled when partly collapsed, but bag-like and bluish when engorged. A form of hypertrophy which has been described by some of the older writers (Solis Cohen and Voltolini), but which is very little mentioned in recent literature, is in the form of a grayish cushion on the surface of the septum close to its posterior edge. The best known growths however are polypi, which spring from the external wall in or above the middle meatus, or from one of the surfaces of the middle or superior turbinals. It is not correct to call these tumors myxomata or fibromata. They are histologically the equivalent of any other form of inflammatory hypertrophy of the mucous membrane. The gelatinous polypi differ from the denser fleshy tumors only by their state of edema. There are however other polypoid tumors which are histologically different, sometimes mixed angiomata, sometimes malignant growths of polypoid shape.

The causes of hypertrophic inflammation are not fully known. In the case of polypi, suppuration, especially of the accessory cavities, is often a demonstrable etiologic factor, but this is not always the case. In some instances it can be observed that acute 
inflammation is the determining condition, which is used in a loose manner and often inappropriately. perhaps best shown by relapses of circumscribed There is, however, a condition which fully leserves hypertrophies in cases under continuous observation. this name. It is characterized by persistent redness In many instances, however, we do not understand and thickening of the mucous membrane of the why chronic inflammation persists and why it leads pharynx and annoys the patient mostly by the ten. to overgrowth of mucous membrane.

Hypertrophy of adenoid tissue differs from the conditions just described, first, by its location, as it occurs only in the pharynx. It presents, moreover, this difference, that while of inflammatory origin at first the enlarged adenoid tissue, at least of the pharyngeal and faucial tonsils, is not necessarily in a continuous state of inflammation. This distinction is based on clinical data; whether it could be demonstrated histologically in adenoid tissue is perhaps questionable. We may thus observe tonsils which are enlarged, but pale, and cause no irritation for long periods of time, while in others the tonsils show by their congestion and liability to acute troubles that they are the seat of persisting inflammation. In the case of the pharyngeal tonsil the same differences may be observed, with the additional criterion that the enlarged but non-inflamed adenoid tissue in this location secretes only the normal clear mucus, while when inflamed it produces pus.

The most striking forms of adenoid hypertrophy are enlargement of the pharyngeal and the faucial tonsils. As far as etiology can be studied clinically it is evident that the over-development of adenoid tissue is the result of acute attacks of nasal inflammation. Where suitable opportunities are offered it can be observed that children with hitherto unimpeded nasal breathing get the characteristic stuffiness due to ade. noid vegetations after severe attacks of coryza. The close relationship between nasal inflammation and the faucial lymphatic tissue is also shown by the not uncommon occurrence of acute tonsillitis from infection of intranasal wounds on the same side. Undoubt. edly a certain predisposition favors the enlargement of the lymphatic tissue. This is suggested by the frequency with which it occurs in certain families and its absence in others. Clinical observation, too, suggests some relationship between hypertrophy of the pharyngeal tonsil and tuberculosis, but lymphatic enlargement is not a tubercular manifestation by itself, although various recent German and French observers have found that enlarged tonsils, both pharyngeal and faucial, may get tubercular by secondary infection, in perhaps one-sixth of all instances. This adenoid tuberculosis can not be recognized clinically, it is entirely latent. The common text-book statement that enlargement of the adenoid tissue is a manifestation of scrofula is very vague. It is more definite to say that some of the scrofulous appearances, the thick lips, the sub-acute catarrh, the eczema under the nose and the suppurative middle ear inflammation are the direct results of enlarged pharyngeal tonsils.

Besides, in the tonsils, hypertrophy of the lymphatic tissue may show itself as enlarged lingual tonsil, as separate follicles on the phayngeal wall, and as thickened columns of adenoid tissue in the posterior lateral corners of the pharynx. In all these locations the hypertrophied tissue is usually in a state of inflammation and the source of decided discomfort. I have, however, not been able to satisfy myself that purulent secretion is produced in any locality except at the roof of the pharynx and by the lymphatic tissue over the root of the tongue.

The term chronic pharyngitis has hitherto been dency to nausen and retching on slight local irritation. It occurs always in connection with some form of chronic nasal suppuration. Another potent etiologic factor, although not an indispensable one in this affection, is the abuse of tobacco and alcohol. It is hence rarely seen in women. On the basis of clinical data, I consider this affection as a diffuse inflammatory hypertrophy of the adenoid tissue which underlies the pharyngeal mucous membrane.

5. Deformitics of the septum may be classified under three heads: $a$, deviations; $b$, articular expansions; c, callus formations.

a. Moderate curvature of the septum is so common that it can not be considered morbid. It is only an unfavorable structural peculiarity. Moreover, uncomplicated deflection of the septum, without additional malformation, causes stenosis only in narrow noses, where there is less than the average width between the two external walls. We know nothing definite concerning the conditions which lead to asymmetry. It is evidently the result of unproportionate growth of the septum and the bones with which it articnlates. The deflection of the curved septum may be either horizontal or vertical, or exist in both planes.

$b$. The most common clinical malformations of the septum are projecting spurs, ledges or ridges, often but erroneously, termed exostoses. According to Zuckerkandl's extensive dissections, they are formed by overgrowth of the articulating surfaces of the various plates which make up the septum. The term articular expansion seems, therefore, an appropriate one. It is especially the strip of cartilage which persists during adult life on the upper border of the vomer, which expands. Being surrounded by bony walls this cartilaginous hypertrophy thus forms the nucleus of a bony projection on the side of the septum, while occasionally secondary ossification of the cartilaginous strip adds to the solidity. The usual site of these crests is along the upper border of the vomer, running in an oblique direction upward and backward toward the sphenoid sinus. A similar formation is found less commonly as a vertical ridge between the quadrangular cartilage and the perpendicular plate of the ethmoid. Such one-sided expansions are not rarely combined with deflection of the septum, which then presents an angular depression on the concave side and a protruding spur on the convexity. This malformation is often mistaken for a healed fracture.

c. Fractures of the septum are not common. It is doubtful whether they are possible without fracture of the bridge of the nose, and unless a characteristic flattening of the bridge is recognizable, the diagnosis of broken septum is questionable. Fracture of the septum usually leaves considerable deformity on account of the displacement and overlapping of the fragments.

The importance of deformities of the septum hinges largely on the stenosis which they cause. This in turn depends on the relative width of the nasal passages. Even when the nose appears normally patent during examination, transient stenosis may still exist during periods of engorgement of the cavernous tissue. But stenosis itself is not a disease. It is only 
an important anomaly which predisposes to the persistence of chronic inflammation.

Irregularities of the septum may play a morbid role even without reducing the capacity of the nasal passage. An irregular septal surface favors ohronic inflammation, perhaps by impeding the flow of morbid secretions. Hence, successful operations of septal deformities often prove of service even where no stenosis existed. They are not rarely the starting point of morbid reflexes, especially when the spurs are sharp, or when they come in contact with the surface of the turbinals.

For these various reasons operative treatment of septal deformities is often indicated and in the case of extensive malformation it is usually satisfactory. But the minor irregularities, and especially the deformities due to moderate septal deviation, are not se easily benefited mechanically. The novice can not be too strongly warned against attempting operations indiscriminately, as many of these fail primarily for mechanical reasons, or produce but a temporary result on account of relapses.

\section{INJURY OF' THE CAVUM PHARYNGEUM; REPORT OF A CASE. \\ BY EMIL ARONSON, M.D. DALLAS, TEXAS.}

Several years ago, while practicing in Europe I received a call to Mr. D., a gamekeeper, who had sustained an injury from the explosion of a gun In the morning Mr. D. had tried his first experiment with his new gun. While the members of his family were looking on they saw him fall to the ground with blood oozing from nose and ear. $\mathrm{He}$ was carried unconscious into the house. After some time he regained consciousness but did not complain of the presence of a foreign body in his throat, yet his family thought it necessary to send for their family physician. This gentleman arrived, examined the throat and stated that except a piece of a tooth which was lodged in the posterior wall of the pharynx, nothing could be found. Promising to return in a day or two to remove that remnant of the tooth he left the house. Other relatives of the injured man, much surprised at the indifference of this gentleman, decided to send for me.

Inspection reveals the following conditions: Upper lip swollen, upper right incisors are missing; some loose fragments are hanging in the alveoli. The palate, the mucosa buccalis, the tongue, uvula and posterior wall of the pharynx are discolored, powderstained and covered with coagulated blood; on the right side of the posterior wall in the region of the constrictor pharyngeus superior and medius is a mass of iudistinct shape and color.

The gun consists of two long tubes which can be attached to each other. After placing the cartridge in one tube, the other tube is screwed on to it. At the end of this long tube the inventor inserted an iron bolt, he put the tube to his mouth and expected that by very forced expiration the bolt could be driven against the cartridge. His calculation failed to prove correct at the first trial. He did not consider that the moment the bolt and cartridge would meet explosive gases would become free, which, if no valve was provided, would drive the bolt back into the mouth of the experimenter.

Although the patient felt perfectly comfortable and there were no symptoms of stenosis, I managed to make out a large, smooth, round, metallic body in the back wall of the pharynx. Trying twice, I failed to grasp this with a long forceps, due to the impossibility of pressing the points of the forceps into the hard iron bolt. I then took a long Langenbeck retractor and using it as a lever removed the bolt, which weighed nineteen grams and was four and a half centimeters long.

I prescribed an antiseptic gargle and directed ice and antipyrin for the intense headache, which appeared in the course of the week. In about ten days the patient was well again. A few conclusive remarks about the clirection of the projectile: The upper incisors were struck first and knocked out. The projectile then changed its direction and passed through the oral cavity to become imbedded in the muscles of the pharynx, where it lodged on one of the vertebræ. It is remarkable and of value in regard to diagnosis, how very little pain was felt by the patient, who for more than twelve hours did not believe there was a foreign body in his throat. This case shows how valuable an objective examination is and teaches us not to rely entirely upon the patient's statements or upon the observations of Schroetter (notably, on "Diseases of the Ear," 1876 and 1882), who says that in such injuries of the pharynx the patients always claim to feel the foreign body in the throat.

\section{DISEASES OF THE EYE CAUSED BY DIS- EASES OF THE NOSE.}

Read before the Chicago Academy of Medicine, December, 1897. BY ALLEN T. HAIGHT, M.D.

PROFESSOR OF OPHTHALMOLOGY CHICAGO EYE, EAR, NOSE AND THROAT COLLEGE; ATTENDING OPHTHALMOLOGIST AND OTOLOGIBT TO THE GERMAN AMERICAN HOSPITAL; MEMLER OF THE AMERICAN MEDICAL ASSOCIATION, ETC. CHICAGO, II.L.

There are no organs of the human body more closely connected than the eye and the nose. No organ suffers more from reflexes than the eye from the nose. The slightest offense to the mucous membrane of the nasal chambers will instantly cause suffering to the eye. Let one come in contact with smoke or other irritants to the Schneiderian membrane and the eye will soon respond to its danger and shed tears. The reason for this is clear, as the eye is directly connected with the nose by the nasal duct, the same mucous membrane, histologically connected, continues to cover the eye lids, the globe and the orbits.

The lachrymal artery with its branch, the anterior ethmoid, supplies blood nourishment to both the lachrymal sac and the nasal duct. The nerve supply is received in common from branches of the ophthalmic nerve and Gasserian ganglion. The lachrymal sac, the dilated part of the nasal duct, is situated in a channel of the nasal process of the superior maxillary and lachrymal bones. Its formation is fibrous and elastic, lined with mucous membrane, continuous with that of the nose. The membrane of the lachrymal sac and canaliculi is lined with pavement epithelium, but in the main duct the epithelium is partly of a cylindric variety. The middle lachrymal organ (Fig. 1) consists of a small double puncta, located from six to eight millimeters from the apex of the inner canthal angle, opening into the corresponding superior and inferior canaliculi, which meet toward the inner canthus in the lachrymal sac.

"The lachrymal secretion is forced through the puncta into the canalicula, then enters the sac and 